\title{
Exploiting differential Wnt target gene expression to generate a molecular biomarker for colorectal cancer stratification
}

\author{
Sam 0 Kleeman (10, ', Viktor H Koelzer, ${ }^{1,2}$ Helen JS Jones, ${ }^{1,3}$ Ester Gil Vazquez, ${ }^{1}$ \\ Hayley Davis @ (1) ${ }^{1}$ James E East, ${ }^{4}$ Roland Arnold, ${ }^{5}$ Martijn AJ Koppens, ${ }^{1}$ \\ Andrew Blake, ${ }^{6}$ Enric Domingo, ${ }^{6}$ Chris Cunningham, ${ }^{3}$ Andrew D Beggs (1) ${ }^{7}$ \\ Valerie Pestinger, ${ }^{5}$ Maurice B Loughrey, ${ }^{8}$ Lai-Mun Wang ${ }_{1}^{9}$ Tamsin RM Lannagan, ${ }^{10}$ \\ Susan L Woods 이, ${ }^{10}$ Daniel Worthley, ${ }^{10} \mathrm{~S}$ :CORT Consortium, Ian Tomlinson 다, ${ }^{5}$ \\ Philip D Dunne (1) , ${ }^{8}$ Timothy Maughan, ${ }^{6}$ Simon J Leedham ${ }^{1}$
}

\begin{abstract}
Additional material is published online only. To view, please visit the journal online (http://dx.doi.org/10.1136/ gutjnl-2019-319126).

For numbered affiliations see end of article.
\end{abstract}

\section{Correspondence to} Professor Simon J Leedham, Wellcome Trust Centre Human Genetics, University of Oxford, Oxford OX3 7BN, Oxfordshire, UK; simonl@well.ox.ac.uk

Received 18 May 2019 Revised 20 August 2019 Accepted 7 September 2019 Published Online First 28 September 2019

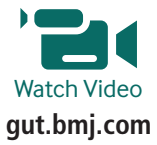

Check for updates

(c) Author(s) (or their employer(s)) 2020. Re-use permitted under CC BY. Published by BMJ.

To cite: Kleeman $\mathrm{SO}$ Koelzer VH, Jones HJS, et al. Gut 2020;69:1092-1103.

\begin{abstract}
Objective Pathological Wnt pathway activation is a conserved hallmark of colorectal cancer. Wnt-activating mutations can be divided into: i) ligand-independent (LI) alterations in intracellular signal transduction proteins (Adenomatous polyposis coli, $\beta$-catenin), causing constitutive pathway activation and ii) ligand-dependent (LD) mutations affecting the synergistic R-Spondin axis (RNF43, RSPO-fusions) acting through amplification of endogenous Wnt signal transmembrane transduction. Our aim was to exploit differential Wnt target gene expression to generate a mutation-agnostic biomarker for LD tumours. Design We undertook harmonised multi-omic analysis of discovery $(n=684)$ and validation cohorts $(n=578)$ of colorectal tumours collated from publicly available data and the Stratification in Colorectal Cancer Consortium. We used mutation data to establish molecular ground truth and subdivide lesions into LI/LD tumour subsets. We contrasted transcriptional, methylation, morphological and clinical characteristics between groups.

Results Wnt disrupting mutations were mutually exclusive. Desmoplastic stromal upregulation of RSPO may compensate for absence of epithelial mutation in a subset of stromal-rich tumours. Key Wnt negative regulator genes were differentially expressed between $\mathrm{LD} / \mathrm{LI}$ tumours, with targeted hypermethylation of some genes (AXIN2, NKD1) occurring even in CIMP-negative LD cancers. AXIN2 mRNA expression was used as a discriminatory molecular biomarker to distinguish LD/LI tumours (area under the curve $>0.93$ ).
\end{abstract}

Conclusions Epigenetic suppression of appropriate Wnt negative feedback loops is selectively advantageous in LD tumours and differential AXIN2 expression in LD/LI lesions can be exploited as a molecular biomarker. Distinguishing between LD/LI tumour types is important; patients with LD tumours retain sensitivity to Wnt ligand inhibition and may be stratified at diagnosis to clinical trials of Porcupine inhibitors.

\section{INTRODUCTION}

The Wnt pathway is critical for intestinal development and adult tissue cell fate determination. ${ }^{1}$ Consistent with this key homeostatic role, epithelial Wnt

\section{Significance of this study}

What is already known on this subject?

- Precision medicine requires simple and accurate stratification of patients in advance of treatment decisions.

- The Wnt pathway can be activated either through constitutive activation of downstream signal transduction (ligand-independent (LI) mutation) or from the disruption of the synergistic R-Spondin axis (ligand-dependent (LD) mutation).

- Preclinical models of LD tumours retain therapeutic sensitivity to ligand inhibition, and small molecule Porcupine inhibitors are in early phase clinical trials.

What are the new findings?

- Wnt ligand-dependent and ligand-independent tumour subgroups exhibit non-overlapping transcriptional, epigenetic, morphological and clinical characteristics.

- We identify a rare tumour subset, termed $R S P O$-high with Wnt disruption predominantly driven by desmoplastic stromal expression of R-Spondin ligands.

- Differential expression of some Wnt negative regulator genes between tumour subgroups is associated with targeted promoter methylation in LD tumours.

- One of these differentially expressed negative regulators, AXIN2, can be used as a discriminatory molecular biomarker of $L D$ tumours with an area under the curve $>0.93$.

signalling is strictly regulated, with activity predominantly restricted to the bottom half of the intestinal crypt where the adult intestinal stem cell niche and the proliferative transit amplifying cells are located. ${ }^{2}$

The canonical Wnt ligands are predominantly expressed by stem cell niche constituents including telocytes, ${ }^{3}$ Gli1+ve fibroblasts ${ }^{4}$ and Paneth cells. ${ }^{5}$ 
Significance of this study

How might it impact on clinical practice in the foreseeable future?

- Currently, identification of solid tumour patient subgroups with LD mutations would require multi-omic analysis which is impractical for routine clinical use.

- Use of AXIN2 as a single gene, mutation-agnostic biomarker on diagnostic biopsies could help stratify patients that might benefit from Wnt ligand inhibition.

These paracrine morphogens are secreted following palmitoylation by the membrane bound O-acyltransferase, Porcupine ${ }^{6}$ and this post-translational modification is required for activity of Wnt ligands. Wnt activation results from the binding of ligand to cognate Frizzled (FZD) and lipoprotein receptor-related protein receptors on the cell surface. Recently, the R-Spondin signalling pathway has emerged as a key, co-evolved pathway for Wnt amplification. In the absence of R-Spondin ligand, cellsurface Wnt FZD receptors are degraded by the activity of the E3 ubiquitin ligases Ring finger protein 43 (RNF43) and Zinc and Ring Finger 3 (ZNRF3). Binding of secreted R-Spondin morphogens to transmembrane leucine-rich repeat-containing $G$ protein coupled receptors 4-6 inhibits RNF43/ZNRF3, disinhibiting Fzd receptors and powerfully amplifying Wnt signalling activity without affecting endogenous canonical Wnt ligand tone. ${ }^{7}$

The key intracellular signal transducer of the canonical Wnt pathway is $\beta$-catenin, encoded by Catenin-Beta1 (CTNNB1). In the absence of Wnt ligand, cytosolic $\beta$-catenin is targeted for degradation by a multi-protein destruction complex consisting of Adenomatous polyposis coli (APC), Axin-like protein (Axin 1/2), Glycogen synthase kinase (GSK3 $\beta$ ) and Casein kinase (CK1 $\alpha)$. Ligand-receptor binding blocks the activation of the destruction complex, releasing $\beta$-catenin which accumulates in the cytosol, translocates to the nucleus and forms a transcriptional complex, activating a large number of Wnt target genes.

In adult intestinal homeostasis, precise control over the polarised gradient of Wnt signalling activity is vital to permit homeostatic cell function, while avoiding the significant pathological consequences of disrupted Wnt activity. Autoregulatory control of the pathway is exerted by negative feedback loops and these control and fine-tune the physiological Wnt rheostat. These loops are rapidly activated following Wnt pathway stimulation, and act at multiple levels of the signalling cascade including: secreted antagonists (eg, secreted FZD-related proteins, ligand palmitoleoylate hydrolases, eg, Notum palmitoleoyl-protein carboxylesterase (NOTUM), receptor inhibitors, eg, Dickkopfrelated protein (DKK), Adenomatosis polyposis coli downregulated 1 (APCDD1) and signal transduction inhibitors, eg, AXIN1/2, Naked Cuticle 1 (NKD1)). Animal models show varying developmental and pathological consequences of Wnt inhibitor knockout, reflecting the context dependency of Wnt signalling function in different cell types. ${ }^{8}$ It is this evolved complexity of the Wnt regulatory network that permits contextdependent amplification or attenuation of Wnt signal activity.

Loss of control of this strictly regulated pathway is associated with pathology in many organs. In the intestine, pathological Wnt activation is a conserved hallmark of intestinal cancer with a spectrum of activating alterations seen in the vast majority of colorectal cancers (CRC). ${ }^{9}$ These include loss-of-function mutations in APC and RNF43 and gain-of-function mutations in RSPO (characterised by gene fusions) and CTNNB1. ${ }^{9-11}$ While
$A P C$ and CTNNB1 alterations drive downstream activation of the Wnt pathway that is independent of Wnt ligand binding (ligand-independent (LI)), RSPO and RNF43 alterations disrupt the synergistic R-Spondin axis and amplify endogenous Wnt ligand signalling (ligand-dependent (LD)). These mutations are almost always mutually exclusive in CRC, which is consistent with previous work demonstrating that an optimal but not excessive level of Wnt activation is considered favourable for tumourigenesis—-the 'just-right' theory. ${ }^{12}$

Interestingly, different Wnt pathway mutations are selectively and preferentially acquired in different polyp subtypes. Thus, conventional tubular and tubulovillous adenomas are characterised by APC or, less commonly, CTNNB1 mutations while serrated polyps such as sessile serrated lesions (SSL) and traditional serrated adenomas (TSA) commonly select for RNF43 mutation or RSPO fusions, suggesting that tumours with these mutations follow a distinct evolutionary trajectory. ${ }^{13}$ Proactive molecular stratification of LD or LI Wnt driver mutations is potentially clinically important; tumours driven by LD Wnt driver mutations retain sensitivity to Wnt inhibition through Porcupine inhibitors or anti-RSPO antibodies. ${ }^{14}$ However, there is no simple biomarker available to identify the LD patient cohort which currently requires undertaking targeted panel/ exome sequencing, together with RNA sequencing for RSPO fusions. Here, we show that differential Wnt negative feedback target gene expression between LD and LI tumours can be exploited to generate a mutation-agnostic, simple, single molecular biomarker to discriminate between tumours, and that this could be used clinically to identify patients with cancers that might be sensitive to preoperative Porcupine inhibitor therapy.

\section{METHODS}

Multi-omic profiling performed in each patient cohort is summarised in online supplementary table 1 . See online supplementary methods for details on data access, sample selection, nucleic acid extraction, quantitative real-time PCR (qRT-PCR), bioinformatic analyses, in situ hybridisation (ISH) and digital pathology.

\section{RESULTS}

\section{Cohort characteristics}

As detailed in table 1.

\section{Driver Wnt pathway alterations are mutually exclusive in colorectal polyps, colorectal tumours and non-colorectal tumours}

To characterise the landscape of Wnt-altering events in CRC and other tumours, we undertook multi-omic analysis of cohorts from the Stratification in Colorectal Cancer (S:CORT) study and publicly available data (methods). The aim was to segregate tumours into two clusters: LI tumours with identifiable mutations in APC or CTNNB1 and LD lesions with RSPO2/3 fusions or RNF43 alterations. APC, RNF43 and CTNNB1 mutations were identified by DNA sequencing. RSPO2 and RSPO3 gene fusions were identified by the presence of specific fusion breakpoint sequences in RNA sequencing reads.

Discovery cohorts included a selected precancerous polyp cohort $(\mathrm{n}=54$ from $\mathrm{S}$ :CORT), deliberately incorporating polyps from across the histological spectrum (clinicopathological features summarised in online supplementary table 2). Mutational analysis identified activating Wnt alterations in 41/54 polyps (76\%) (figure 1A, online supplementary tables 3 and 4 ). The 13 lesions without detectable mutation were non-dysplastic 
Table 1 Cohort characteristics for the seven cohorts of samples included in this study

\begin{tabular}{lllllllll}
\hline Cohort & & Origin & Type & Preparation & Sample size & Pathology & Mean age & M:F ratio \\
\hline Discovery & Polyps & Internal (S:CORT) & Biopsy & Fresh/frozen & $\mathrm{n}=54$ & Adenoma & 67.0 & 1.00 \\
& $\mathrm{~A}(\mathrm{DC}-\mathrm{A})$ & $\mathrm{TCGA}^{9}$ & Resection & Fresh/frozen & $\mathrm{n}=618$ & Carcinoma (colon, rectum) & 66.4 & 1.15 \\
& $\mathrm{~B}(\mathrm{DC}-\mathrm{B})$ & Genentech $^{11}$ & Resection & Fresh/frozen & $\mathrm{n}=66$ & Carcinoma (colon) & NA & NA \\
& Pancancer & TCGA $^{17}$ & Resection & Fresh/frozen & $\mathrm{n}=10542$ & Carcinoma (non-colorectal) & 59.1 & 0.92 \\
Validation & $\mathrm{A}($ Val-A) & Internal (S:CORT) & Resection & FFPE & $\mathrm{n}=348$ & Carcinoma (colon, rectum) & 63.5 & 1.79 \\
& $\mathrm{~B}($ Val-B) & Internal (S:CORT) & Biopsy & FFPE & $\mathrm{n}=230$ & Carcinoma (rectum) & 67 & 1.71 \\
Clinical application & & Internal & Resection & FFPE & $\mathrm{n}=63$ & Carcinoma (rectum) & 70.4 & 1.63 \\
\hline
\end{tabular}

FFPE, formalin-fixed paraffin embedded; NA, not available; S:CORT, Stratification in Colorectal Cancer.

SSLs-this is consistent with evidence that SSLs acquire Wnt alterations at an advanced stage, as part of progression to dysplasia. ${ }^{15}$ All Wnt alterations identified were mutually exclusive. In line with previous findings, ${ }^{16}$ LD alterations were only identified in TSAs and SSLs, whereas LI alterations were seen across all histological polyp types.

We applied the same stringent mutational analysis to two CRC discovery cohorts (discovery cohort A from TCGA and discovery cohort B from Genentech) incorporating 684 colorectal tumours. Functionally relevant, activating Wnt alterations were identified in $431 / 684(63 \%)$ tumours (figure 1A, online supplementary tables 3 and 4). In this large tumour cohort, we identified just 14/431 (3\%) tumours with more than one detectable Wnt driver alteration.

To assess the spectrum of Wnt drivers in tumours from other primary sites, we undertook mutational analysis across the TCGA pan-cancer cohort of 10542 non-colorectal tumours ${ }^{17}$ and identified Wnt mutations in 203/10 542 (2\%) tumours (figure 1A, online supplementary tables 3 and 4). The most common primary sites for tumours with Wnt disrupting alterations were endometrium, liver and stomach. Consistent with results in our colorectal cohorts, only 4/203 (2\%) of non-CRC tumours contained more than one Wnt driver. Together, this mutual exclusivity suggests strong selection pressure against acquiring more than one Wnt driver mutation.

\section{Stromal overexpression of R-Spondin as a novel driver alteration in colorectal cancer}

To internally validate our fusion calling we determined normalised RSPO2/3 expression in samples with and without $R S P O$ fusions. In the polyp cohort, we found that all polyps with outlier RSPO expression (defined as z-score $\geq 2$ ) had detectable RSPO fusions (online supplementary figure 1). In contrast, in the discovery cohort, we identified a subset of tumours (termed RSPO-high, $\mathrm{n}=16 / 684$ ) with outlier expression of $R S P O 2 / 3$ despite absence of detectable fusion mutation (online supplementary figure 1$)$. The majority of these samples (10/16) had no other identifiable Wnt alterations. Homeostatically, RSPO expression exclusively from muscularis mucosae fibroblasts maintains the intestinal crypt stem cell niche, so we reasoned that tumour microenvironmental RSPO expression could compensate for the absence of epithelial Wnt mutations in RSPO-high tumours. Consistent with a stromal R-Spondin source, analysis of gene expression profiles in RSPO-high versus RSPO-fusion tumours showed that the former exhibit significant upregulation of a validated cancer-associated fibroblast gene signature ${ }^{18}$ (figure $1 \mathrm{~B}, \mathrm{p}=9.04 \times 10^{-8}$ ), and that $10 / 12$ RSPO-high tumours with available consensus molecular subtype (CMS) classification were CMS4 (mesenchymal) subtype tumours (figure 1C). In addition, blinded expert histopathological review of digital slides identified trends towards increased tumour budding and reduced glandular formation in RSPO-high versus RSPO-fusion lesions (online supplementary figure 1B).

As these discovery sets were derived from publicly available datasets, we had no tissue access to check on-slide RSPO cell compartment expression. We therefore collated a cohort of tumours with available tissue sections derived from both a clinical application cohort of rectal tumours $(n=63$, table 1$)$ and validation cohort A ( $\mathrm{n}=348)$. Tumours with outlier RSPO3 expression were profiled using both targeted RSPO3 RNA sequencing to identify fusion genes and ISH to visualise the origin of the RSPO3 signal in these samples. We identified six tumours with outlier RSPO3 expression, four of which had detectable RSPO3 fusions. The RSPO-fusion tumours exhibited a tissue compartmental shift in expression with epithelial localisation of transcripts, whereas RSPO-high tumours exhibited marked upregulation of RSPO3 expression from the desmoplastic stroma (figure 1D, online supplementary figure 2). Finally, ISH on a subset of tumours with non-outlier RSPO3 expression $(n=20)$ demonstrated qualitative correlation between expression and stromal staining, altogether suggesting that RSPO3 stromal expression in RSPO-high tumours reflects an extreme perturbation of constitutive stromal RSPO3 expression (online supplementary figure 2).

In summary, RSPO-high tumours characterise a rare tumour subset, with Wnt disruption predominantly driven by desmoplastic stromal overexpression of R-Spondin ligands. We incorporated this subset of tumours in our LD cluster for further analysis. After including this RSPO-high cohort as potential LD tumours, the total number of tumours with detectable LD alterations was $75 / 684(11.3 \%)$.

\section{Distinct regulation of endogenous Wnt negative feedback loops in ligand-dependent tumours}

Having established the molecular ground truth, we segregated tumour samples in the discovery cohort into LD (RSPO-fusions, RSPO-high and RNF43 mutations, $\mathrm{n}=64$ ) and LI (APC or CTNNB1 mutations, $\mathrm{n}=347)$ subsets. Samples with concurrent LD and LI alterations $(n=9)$ were excluded to minimise the potential for misclassification (figure 2A). In light of the specific polyp type predilection and mutual exclusivity of different Wnt mutations in colorectal tumourigenesis, we hypothesised that, rather than being interchangeable, LD and LI alterations drive distinct patterns of Wnt pathway activation. To assess this, we performed differential gene expression analysis between LD and LI lesions in our discovery cohorts (online supplementary figure 3A) and undertook gene-set enrichment analysis, incorporating four curated subsets of Wnt target genes (global Wnt responsive, stem cell, proliferative and negative regulator (NR) genes, detailed in online supplementary table 5). While there was no evidence of significant enrichment of global or proliferative Wnt targets in either cohort, there was significant enrichment of NRs (normalised enrichment score $(\mathrm{NES})>2.02, \mathrm{p}<0.003$ ) 

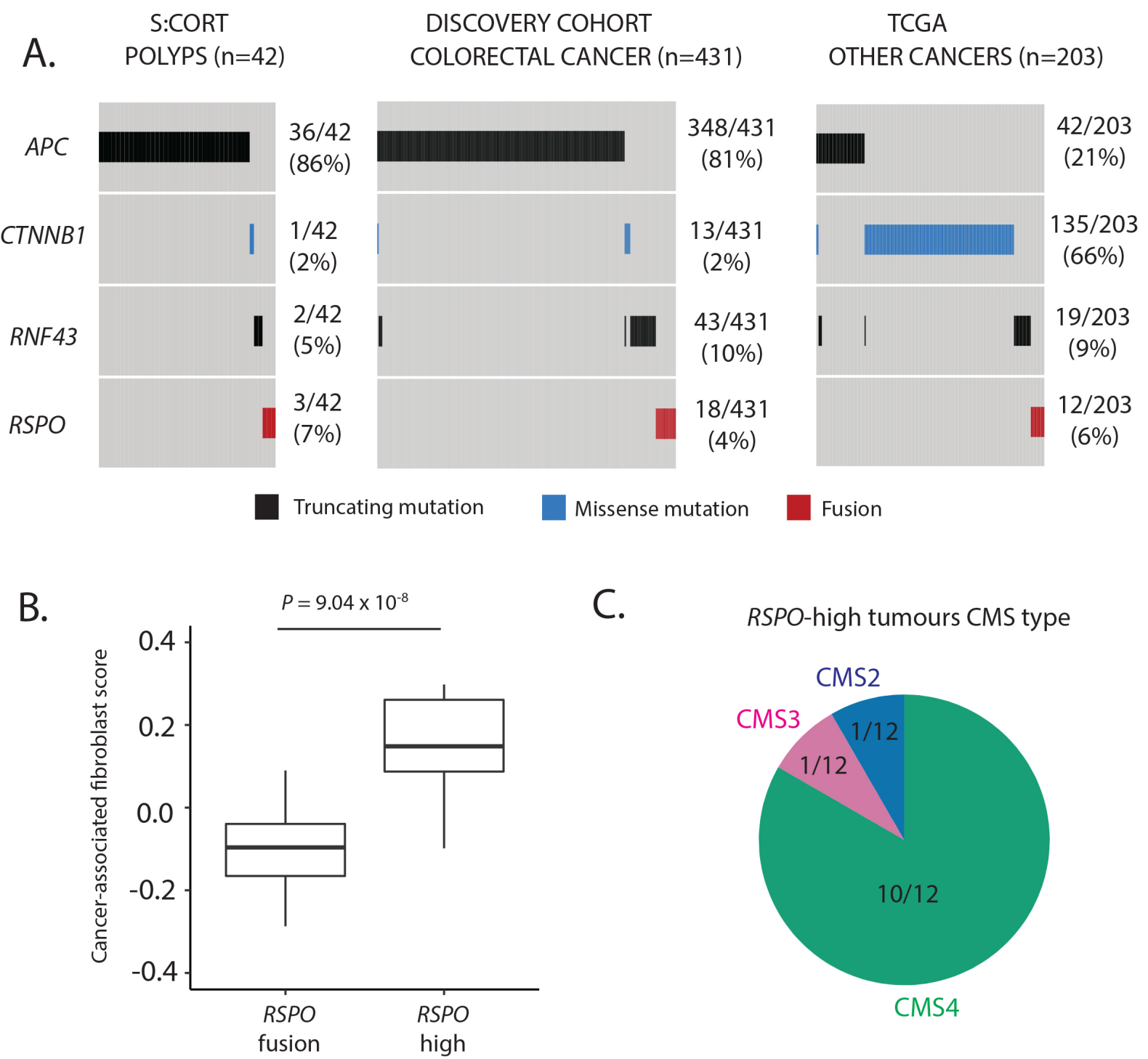

D.

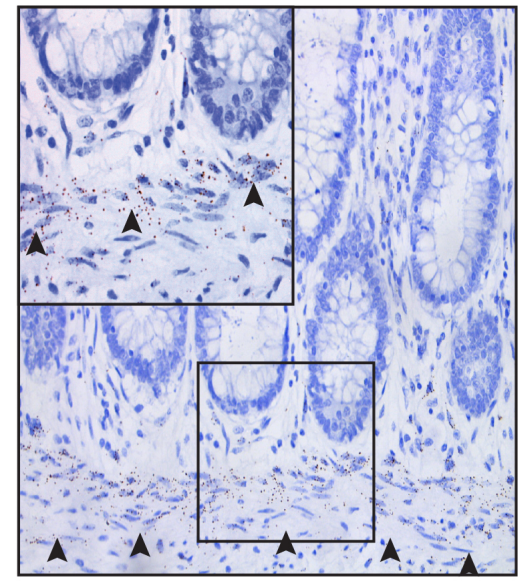

Normal colon

R-SPONDIN3 ISH

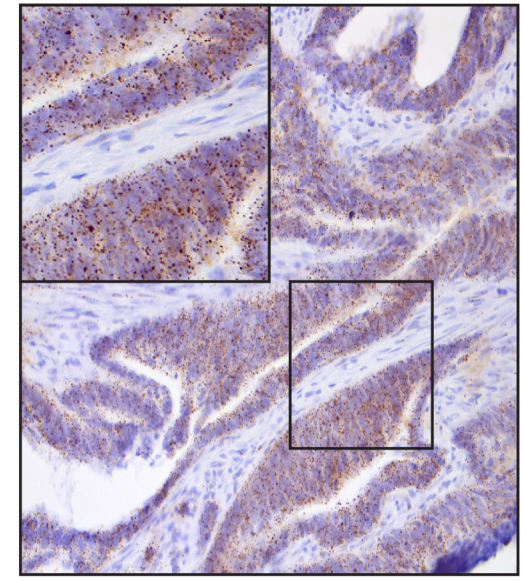

RSPO-fusion tumour

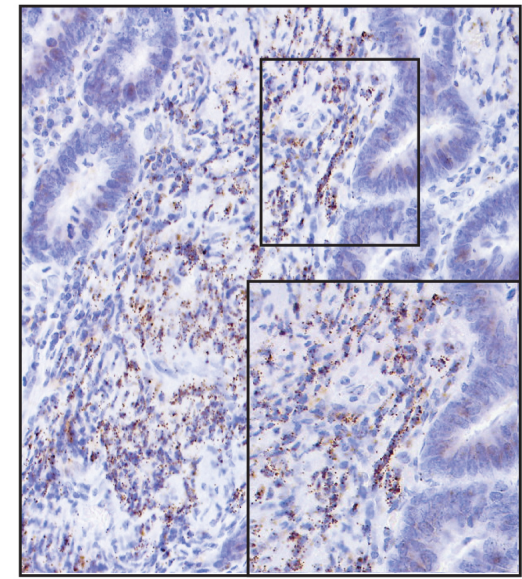

RSPO-high tumour

Figure 1 Wnt mutation burden and RSPO-high tumours. (A) Oncoprint of identifiable Wnt disrupting mutation distribution in the Stratification in Colorectal Cancer (S:CORT) polyp cohort, TCGA CRC cohort and TCGA solid cancer cohorts. Only tumours with detectable Wnt alterations are displayed. (B) Comparison of transcriptome-based cancer-associated fibroblast score in RSPO-fusion and RSPO-high tumours. (C) Consensus molecular subtype (CMS) of RSPO-high tumours. (D) RSPO3 in situ hybridisation (brown spots) exclusively from the muscularis mucosae in normal human colon, with aberrant epithelial expression in an RSPO3-fusion tumour and upregulated stromal expression in an RSPO3-high tumour. 
i) Global Wnt target genes

A.

NES $-0.46 \quad P=1.00$

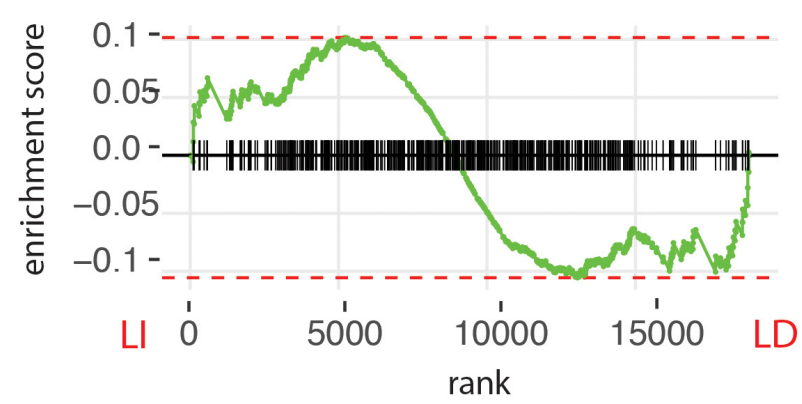

ii) Intestinal stem cell Wnt target genes

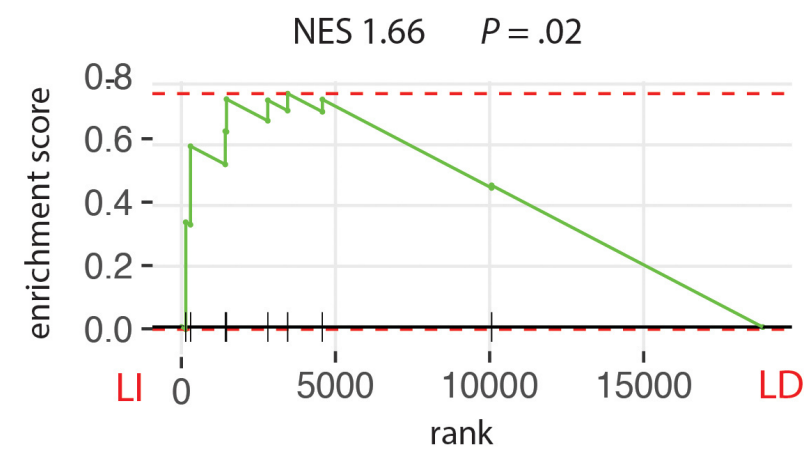

Discovery cohort A - TCGA

iii) Proliferative cell Wnt target genes

NES $1.44 P=.09$

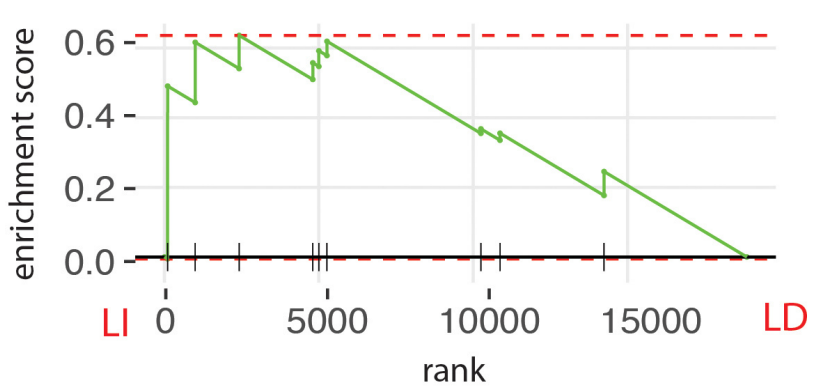

iv) Wnt negative regulator genes

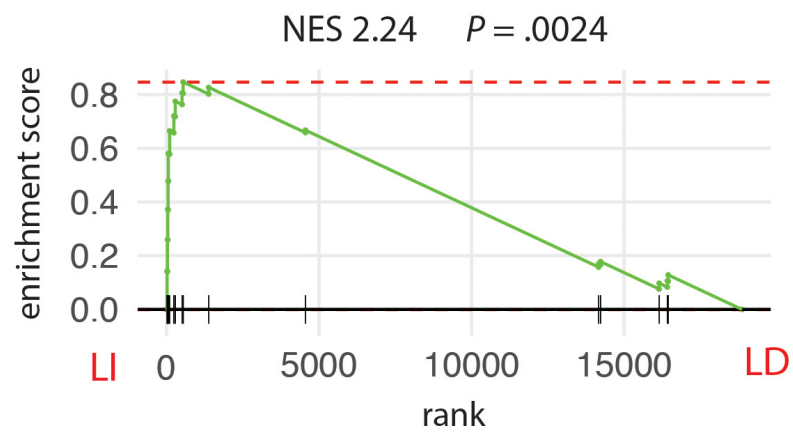

B.

NKD1

$A P C D D 1$

NOTUM

DKK4
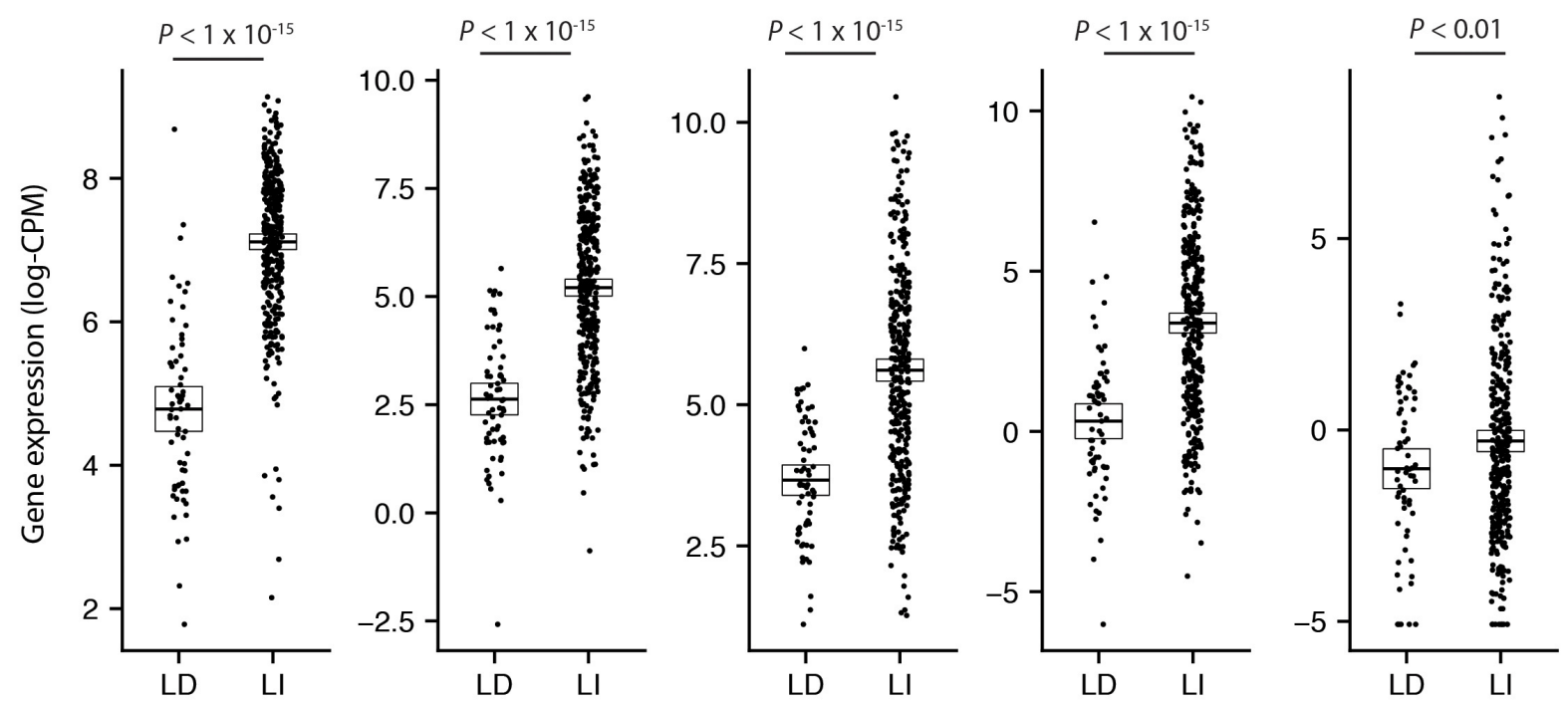

Discovery cohorts

Figure 2 Differential expression of Wnt target genes. (A) Gene set enrichment analysis (GSEA) was performed on discovery cohort A (TCGA) to assess differential expression of i) Global Wnt responsive genes ii) Crypt base columnar stem cell Wnt targets iii) Proliferative cell Wnt targets iv) Wnt negative regulator genes, between ligand-independent (LI) and ligand-dependent (LD) tumours. (B) Gene expression of five leading edge negative regulator (NR) genes between LD and LI tumours in the combined discovery cohorts (log-CPM). NES, Normalised enrichment score. 
and to a lesser extent, stem cell targets (NES $>1.53, \mathrm{p}<0.04)$ in LI tumours (figure 2A, online supplementary figure $3 \mathrm{~B}$ ). Analysis of leading-edge genes within the NR signature identified five consensus differentially expressed NRs in the two discovery cohorts, all expressed at a significantly higher level in LI versus LD tumours-AXIN2, NKD1, APCDD1, NOTUM and DKK4 (figure 2B).

\section{Hypermethylation of endogenous negative regulators in ligand-dependent tumours}

Previous studies have identified evidence of hypermethylation of Wnt NRs, such as AXIN2 and DKK1, in serrated lesions. ${ }^{19}$ As a result, we hypothesised that differential expression of Wnt NRs could be driven by differential methylation. To investigate this, we undertook methylation analysis of tumours in discovery cohort A $(n=440)$ - the only discovery cohort with available methylation data. We identified 26 differentially methylated probes (DMPs) annotated to four of the five differentially expressed Wnt NR genes (AXIN2, NKD1, APCDD1, NOTUM) (online supplementary table 6); 25/26 of these probes were significantly hypermethylated (online supplementary table 6), with significantly elevated mean beta values for each of the four NR genes in LD versus LI tumours (figure 3A, online supplementary figure 4). To understand whether this signal was derived entirely from aberrant methylation in $\mathrm{CpG}$ island methylator phenotype (CIMP) tumours, we determined mean beta value for each NR in both CIMP+ve and CIMP-ve LD and LI tumour subsets. Interestingly, while hypermethylation of NOTUM and $A P C D D 1$ was associated with CIMP positivity (online supplementary figure 4), there was no significant difference in methylation of AXIN2 and NKD1 between CIMP+ve and CIMP-ve tumours, indicating CIMP-independent selective methylation of these genes (figure 3B). Consistent with a functional impact of hypermethylation, AXIN2 and NKD1 exhibit significant and near-linear anticorrelation between methylation and normalised gene expression (figure $3 \mathrm{C}, \mathrm{r}=-0.68$ and $\mathrm{r}=-0.72$ respectively, online supplementary table 7). In contrast, NOTUM and APCDD1 exhibit a bimodal distribution of methylation beta value, implicating a role for alternative regulatory mechanisms (online supplementary figure 4). Together these data suggest that hypermethylation of endogenous Wnt NRs, especially AXIN2 and NKD1, may act in a synergistic fashion with LD Wnt disrupting mutations.

\section{Application of $A X I N 2$ as a clinical biomarker for ligand- dependent tumours}

Having demonstrated consistent, differential expression of Wnt NRs, we hypothesised that we could exploit these discriminatory differences to identify and validate a simple, mutationagnostic biomarker to identify patients with LD tumours that could potentially benefit from Porcupine inhibition. First, we applied receiver operating characteristic (ROC) curve analysis to the LD $(n=64)$ and LI $(n=347)$ subsets within the discovery cohort to determine the diagnostic utility of each consensus NR (online supplementary table 8). This analysis showed that AXIN2 demonstrated superior diagnostic performance with area under the curve (AUC) of 0.93 (95\% CI 0.89 to 0.97 , figure 4A). Using an AXIN2 expression threshold to maximise the sum of sensitivity and specificity, this translated to sensitivity and specificity of $94.5 \%$ and $78.5 \%$, respectively (threshold $=5.75$ log-counts per million/CPM).

Next, to validate the accuracy of AXIN2 as a feasible biomarker for identification of LD tumours, we turned to two validation cohorts (Val-A/Val-B) derived from the S:CORT study (table 1). Using molecular ground truth data, each cohort was stratified into three subsets: LD, LI or unclassified (termed excluded (EX), figure 4B,C). In both cohorts, differential AXIN2 expression was discriminatory between LD and LI tumours while EX tumours exhibited variable AXIN2 expression (figure 4B,C). ROC curve analysis in these validation sets confirmed the LD/LI discriminatory capacity of AXIN2 expression with AUC of $0.95(95 \%$ CI 0.89 to 1.00 ) in Val-A (figure 4B) and 0.97 (95\% CI 0.93 to 1.00 ) in Val-B (figure 4C). Using an AXIN2 expression threshold to maximise the sum of sensitivity and specificity, this translated to sensitivity and specificity of $92.2 \%$ and $90.4 \%$ in Val-A (threshold $=5.58$ log-arbitrary units) and $86.1 \%$ and $100 \%$ in Val-B (threshold $=5.57$ log-arbitrary units).

To further evaluate AXIN2 as a tumour biomarker, we wanted to assess both qRT-PCR and immunohistochemistry (IHC) as alternative approaches for scoring AXIN2 expression in a realistic clinical setting. We extracted RNA from one formalin-fixed paraffin embedded (FFPE) section per lesion from our clinical application cohort $(n=63)$, and immunohistochemically stained a subsequent section for AXIN2 protein. RNA was assayed using both 3'RNA-sequencing and qRT-PCR using the Fluidigm platform. In our hands, only 31/63 of these FFPE samples passed stringent quality control for 3'RNA-sequencing (aligned reads $>75 \%$ ) in contrast to $62 / 63$ samples for qRT-PCR. There was robust correlation between AXIN2 scored by qRT-PCR and 3'RNA-sequencing $\left(\mathrm{n}=31, \mathrm{r}=0.75, \mathrm{p}=1.73 \times 10^{-6}\right.$, online supplementary figure 5). Next, we scored AXIN2 IHC staining on available sections $(\mathrm{n}=53)$ using two complementary approaches: automated scoring using the HALO digital pathology platform and manual scoring by expert histopathologist in a random subset of tumours $(n=27)$. Across the cohort, we identified weak correlation between qRT-PCR metrics of AXIN2 mRNA expression and manual scores of AXIN2 IHC $(r=0.35, p=0.077$, online supplementary figure 5), while there was no significant correlation with automated scores of AXIN2 IHC $(\mathrm{p}>0.29)$. However, the four tumours with known LD status (RSPO-fusion $\mathrm{n}=3$, RSPO-high $\mathrm{n}=1$ ) had comparably low manually scored AXIN2 protein expression (online supplementary figure 5). In summary, qRT-PCR for AXIN2 is a feasible approach to assay AXIN2 expression in a situation of limited tissue availability, while IHC staining is insufficiently sensitive to consistently reflect differences in AXIN2 transcriptome expression.

\section{Ligand-dependent disease positioning in colorectal cancer}

In light of evidence that LD tumours derive from distinct precursor lesions and have different Wnt pathway dynamics, we hypothesised that there would be conserved clinicopathological differences between LD and LI tumours, and detectable differences between subsets with distinct LD alterations. To investigate this, we pooled tumours with available clinicopathological annotations (discovery cohort $\mathrm{A}$ and validation cohort $\mathrm{A}, \mathrm{n}=635$ ). We then contrasted demographics (age, sex, tumour location), molecular pathology (BRAF/KRAS mutation status, microsatellite instability, CMS subtype), computationally scored histopathology (mucin, stroma, tumour and glandular area per slide) and prognostic (overall survival, disease-free survival (DFS)) metrics for each subtype.

In comparison to LD tumours, LI tumours were significantly enriched for left-sided tumours (sigmoid and rectum), KRAS mutations and CMS2 (canonical) classification, consistent with conventional lesion molecular pathway (figure 5A,B, online supplementary table 9). Analysis of prognostic data 

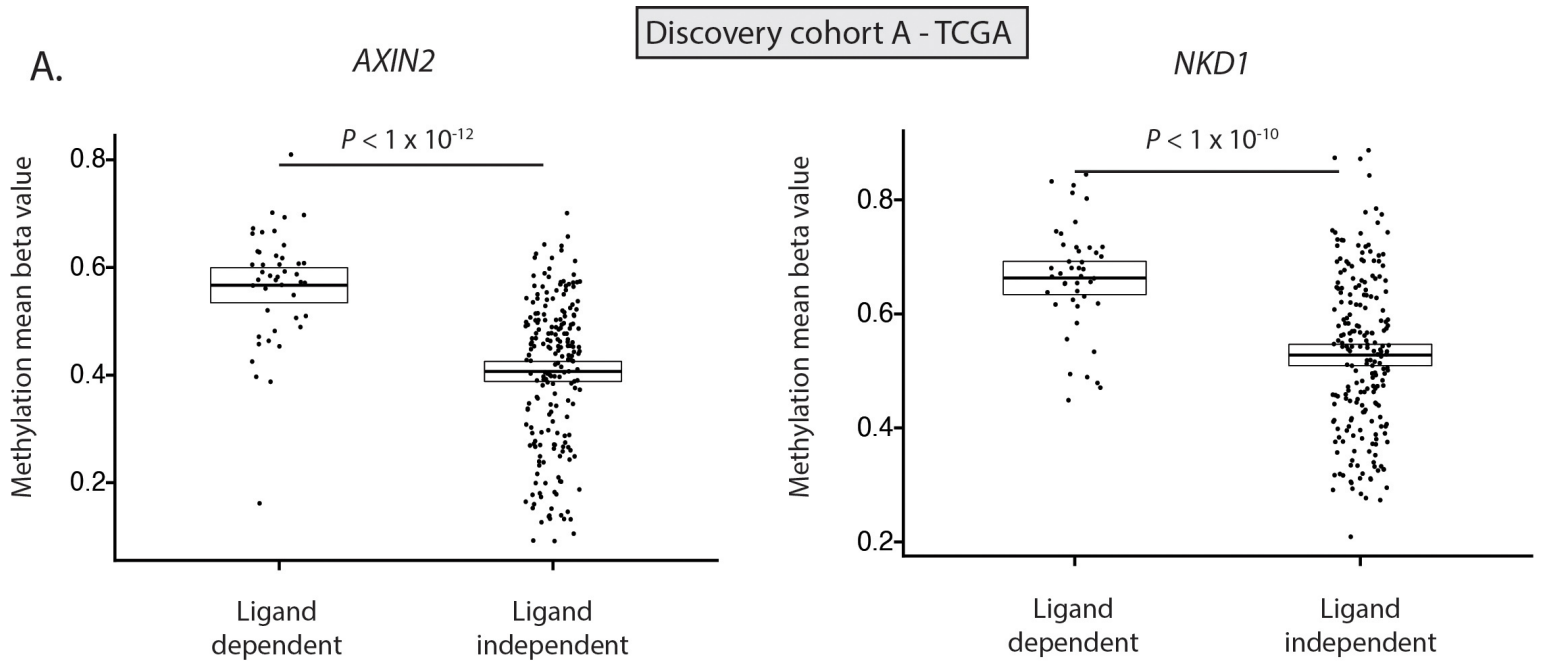

B. AXIN2
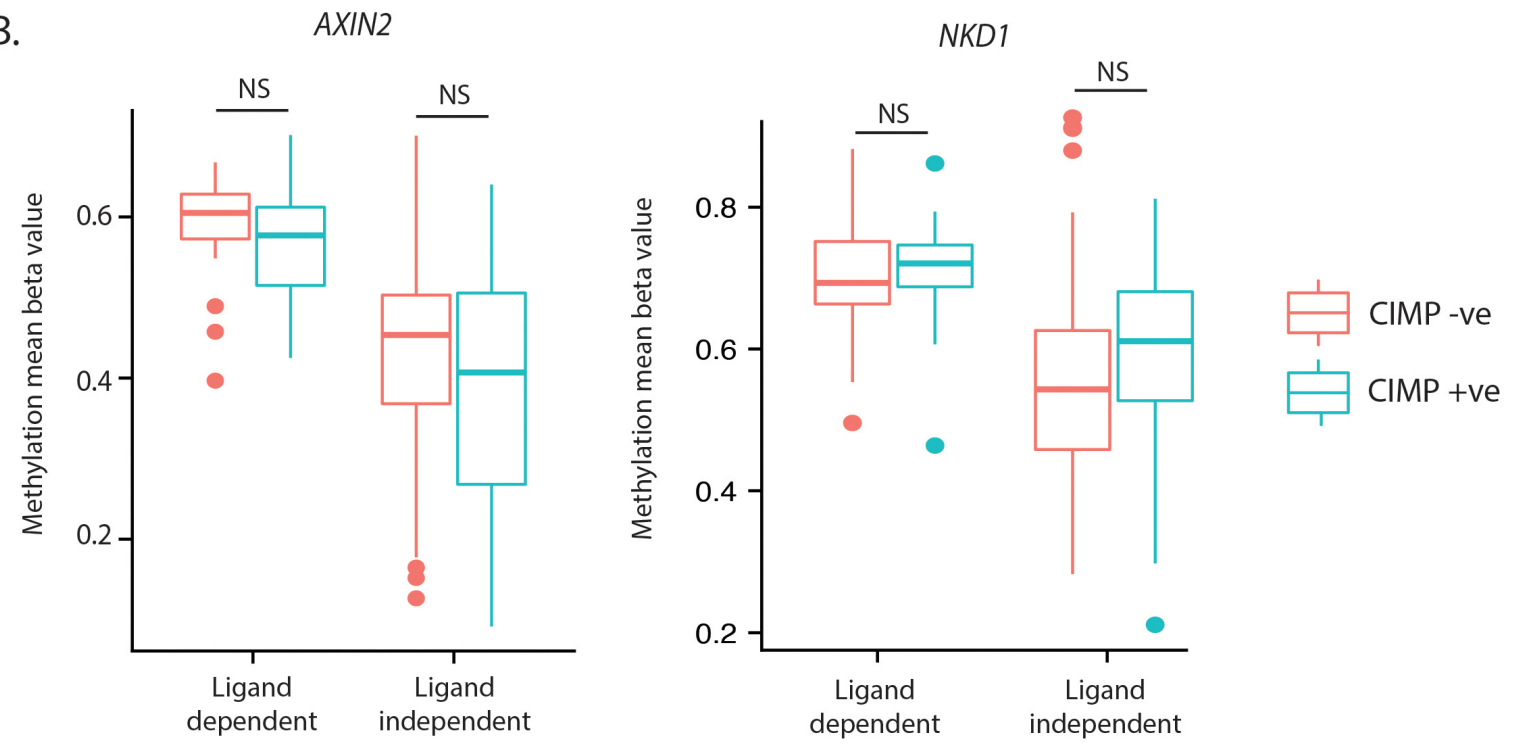

C.

AXIN2

$$
r=-0.68, P<1 \times 10^{-15}
$$

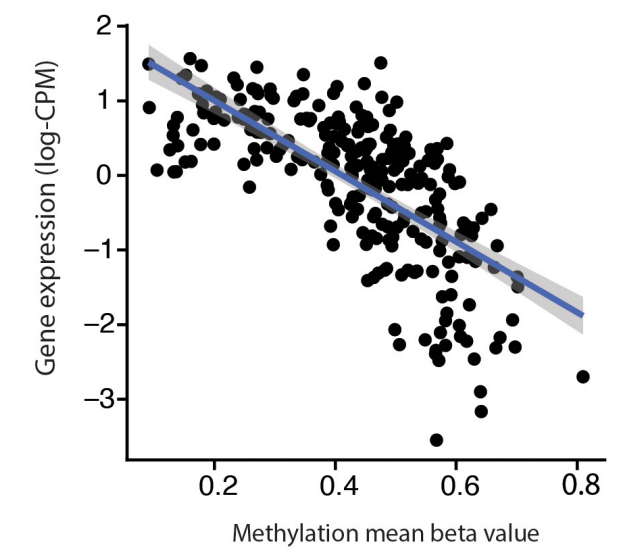

NKD1

$$
r=-0.73, P<1 \times 10^{-15}
$$

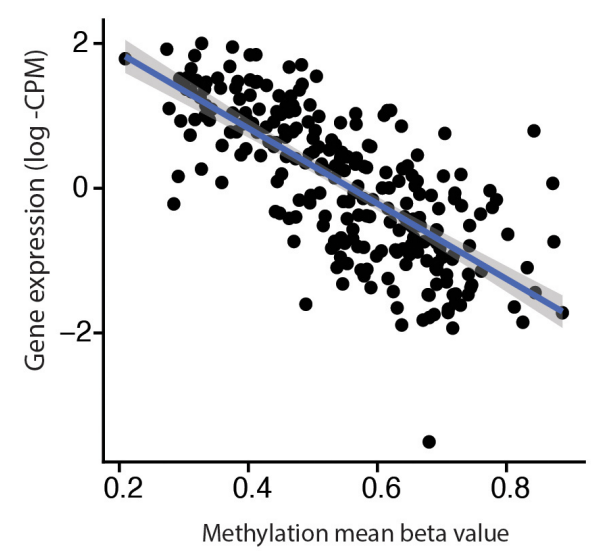

Figure 3 Methylation of key negative regulator genes. (A) Methylation (mean beta value) of AXIN2 and NKD1 showing differential methylation of Wnt NR genes between LD and LI tumours. (B) Methylation (mean beta value) of AXIN2 and NKD1 shows no significant difference between CpG island methylator phenotype (CIMP) positive (blue), and negative (red) LD and LI tumours. (C) Significant anticorrelation between AXIN2 and NKD1 normalised gene expression (log-counts per million/CPM) and gene methylation (mean beta value). All data from discovery cohort A (TCGA). 
A i.

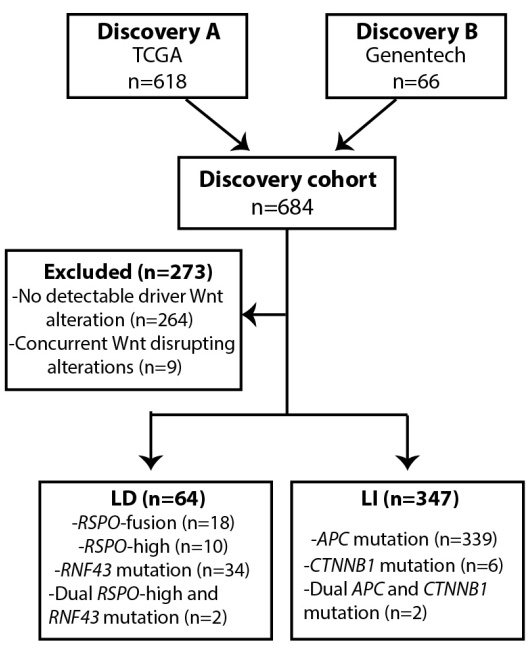

Bi.
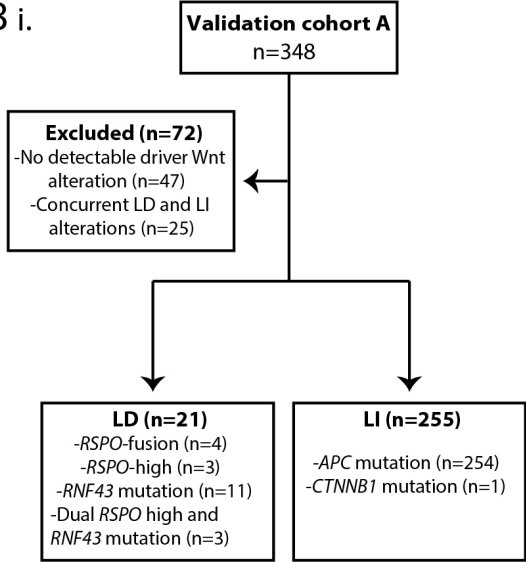

Ci.

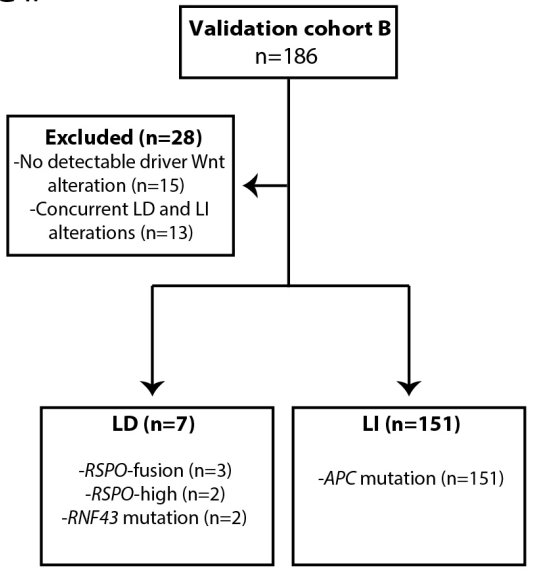

ii

\section{Discovery cohort}

iii
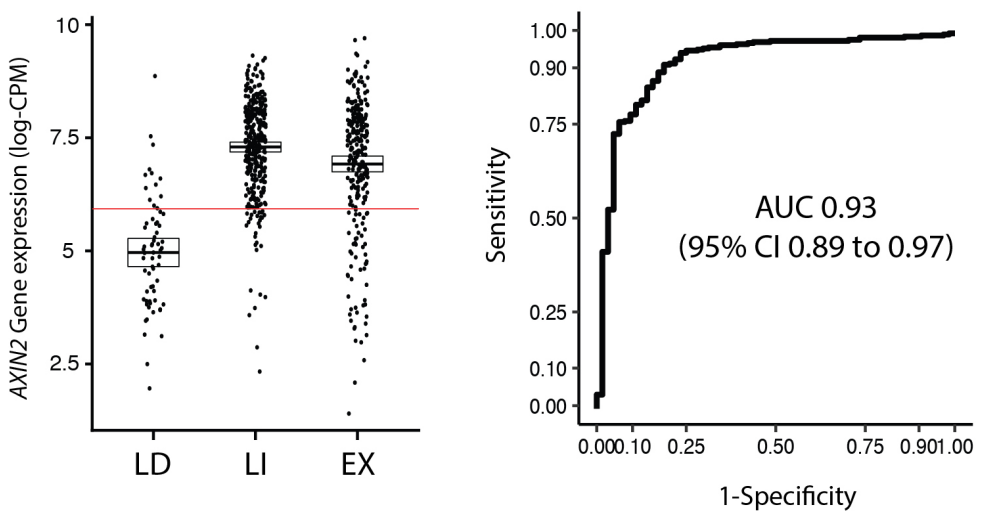

Figure 4 AXIN2 as a clinically discriminatory biomarker. (A) Combined discovery cohorts (TCGA and Genentech). (B) Validation cohort A (S:CORT). (C) Validation cohort B (S:CORT). i) Flow diagram showing exclusion criteria. ii) Differential AXIN2 mRNA expression between LD and LI tumours. iii) Receiver operating characteristic (ROC) curve to assess diagnostic performance of AXIN2 expression. AXIN2 thresholds shown by red lines in combined discovery cohorts, validation cohort A and validation B were $5.75 \log$-CPM, $5.58 \log$-arbitrary units and 5.57 log-arbitrary units respectively. 
identified a non-significant trend towards worse DFS in LI tumours $(\mathrm{HR}=1.42, \mathrm{p}=0.0594)$. LD tumours were also substratified into RSPO-fusion, RSPO-high and RNF43-mutant subsets. To minimise misclassification of tumours, samples with both outlier RSPO2/3 expression and RNF43 mutations $(\mathrm{n}=6)$ were excluded leaving a total of 68 tumours. RNF43-mutant tumours were significantly enriched for right colonic distribution, $B R A F$ mutations, microsatellite instability and CMS1 (MSI immune) classification, consistent with predominantly sessile serrated lesion aetiology (online supplementary table 10). Conversely, $50 \%$ of RSPO-fusion tumours were located distal to the sigmoid, with a lower frequency of CMS1, suggesting enrichment of tumours progressing from traditional serrated adenoma precursors (online supplementary figure $5 \mathrm{C}$ ).

Unexpectedly, using automated segmentation of available scanned tumour sections $(n=194)$ we found that computationally scored mucin area was significantly increased in LD tumours $\left(\mathrm{p}=2.02 \times 10^{-5}\right.$, figure $\left.5 \mathrm{C}\right)$. To validate this finding, we extracted published $^{20}$ manually scored mucin area data for a subset of tumours in DC-A $(n=246)$ and confirmed this metric is also markedly increased in LD tumours $\left(p=2.40 \times 10^{-5}\right.$, figure 5D). To assess the degree to which mucin area can distinguish between LD and LI tumours, we performed ROC curve analysis for each mucin metric. This demonstrated AUC of 0.78 (95\% CI 0.70 to $0.85)$ for computationally scored mucin and AUC of $0.75(95 \%$ CI 0.66 to 0.83 ) for manually scored mucin.

\section{DISCUSSION}

The ability to segregate colorectal tumour patients into clinically meaningful subsets is important to identify patients suitable for targeted therapies and allows comparison and correlation of molecular, morphological and clinical phenotypes. Wnt pathway disruption through selected (epi)genetic mutation is an obligate requirement in almost all CRCs and is common in many other types of solid tumours. However, accurate stratification of patients and successful therapeutic manipulation of pathological Wnt signalling has proven challenging. The majority of colorectal tumours are Wnt ligand-independent, activating the pathway constitutively, through mutation of the intracellular signal transduction machinery. However, a significant subset select for epithelial mutations affecting the synergistic $R$-Spondin axis, rendering them sensitive to therapeutic manipulation of canonical ligand expression. Here, we have also identified a small, but potentially interesting cohort of tumours that lack epithelial Wnt driver mutations altogether-instead profoundly upregulating $R$-Spondin signalling from the tumour stroma. This suggests that stromal compartment expression of Wnt ligands can compensate for absence of selected epithelial mutation in these predominantly aggressive, mesenchymal-subtype (CMS4), 'RSPO-high' tumours. To identify patients with convergent epithelial or stromal LD Wnt disruption, we demonstrate variable expression of Wnt responsive genes in CRC subsets, and use this to highlight differential AXIN2 expression as a simple, discriminatory and mutation-agnostic molecular biomarker.

The mutual exclusivity of LD and LI mutations suggests that activation of either the R-Spondin or downstream canonical axis of Wnt signalling alone is sufficient for tumourigenesis. Multiple Wnt driver mutations result in excessive Wnt disruption and are selected against the 'just-right' hypothesis. However, despite this mutual exclusivity, Wnt disrupting mutations are not necessarily equivalent with respect to downstream target gene activation, with differences in Wnt responsive gene enrichment between LD and LI tumours. The Wnt responsive gene set that showed maximal divergence was 'NRs', with five significantly differentially expressed genes between LD and LI tumours. These important autoregulatory genes are rapidly responsive to $\mathrm{Wnt}$ stimulation and act in parallel, at multiple pathway levels to control the intensity and/or duration of the signal. Expression divergence in these tumour subsets may be partly explained by epigenetic regulation, with hypermethylation of NRs in some LD tumours. Gene methylation correlates with low transcript expression, and Aza-C demethylation treatment restores AXIN2 expression in a dose-dependent fashion in CRC cell lines. ${ }^{21}$ Interestingly, epigenetic suppression of two key Wnt NRs (AXIN2, NKD1) in LD tumours can occur independently of the $\mathrm{CpG}$ island methylator phenotype, where promoter methylation is globally dysregulated. ${ }^{22}$ This suggests that targeted, rather than stochastic methylation of Wnt regulatory genes may occur in these lesions. This is biologically plausible. Wnt activation in LD tumours is the consequence of increased flux through an otherwise intact intracellular signalling pathway. Physiologically relevant and functioning negative feedback loops would constrain pathological LD Wnt signalling, so epigenetic downregulation of involved NR genes would be advantageous in lesions progressing down a LD molecular pathway. In contrast, the constitutive, intracellular activation of Wnt signalling through APC or CTNNB1 mutation in LI tumours, renders upstream Wnt NRs functionally redundant and uncouples appropriate feedback loop equilibrium (figure 6). The mechanistic explanation for these observations needs further detailed functional research, but the differential methylation and expression of key Wnt target genes across the spectrum of LD and LI tumours should be considered when using genes like AXIN2 as a surrogate measure of global Wnt activation.

LD tumours retain sensitivity to Wnt ligand inhibition with Porcupine inhibitor ${ }^{14}$ or anti-RSPO3 antibody therapy ${ }^{23}$ resulting in regression and differentiation of human RSPO-fusion tumours in patient-derived xenograft models. Our analysis across tumour types has detected LD Wnt alterations in colorectal (10\%), stomach $(2 \%)$, endometrial $(1 \%)$ and pancreas $(1 \%)$ tumours while recent work has identified a subset of hepatocellular carcinomas (1\%) with RSPO2 gene fusions. ${ }^{24}$ Detection of these alterations at diagnosis might allow these patients to benefit from chemotherapeutic Wnt ligand suppression. Using current techniques, LD tumour discrimination requires genetic and transcriptomic analysis of all tumours to identify Wnt disrupting mutations and RSPO-fusion transcripts. This is impractical for routine diagnostic assessment. We have exploited the differential expression of Wnt responsive genes across the tumour spectrum to identify a molecular biomarker that discriminates LD/ LI lesions at the target gene level. AXIN2 was the most discriminant single biomarker, with AUC for mRNA expression $>0.93$ in three independent cohorts. Quantitative assessment of AXIN2 mRNA expression was unaffected by the type of sequencing technique (PolyA RNA seq, 3'RNA seq or qRT-PCR), and could be assessed with high discriminatory yield from diagnostic endoscopic biopsy samples in a validation cohort. This supports potential clinical application of the biomarker to stratify patients from endoscopic samples at the point of diagnosis, and in advance of treatment decisions. There was limited correlation between differential RNA expression and the heterogenous IHC staining of AXIN2 protein in our clinical application samples. This may be related to tissue processing variability or antibody insensitivity but known post-translational regulation and altered degradation of AXIN2 protein could also contribute. ${ }^{25}$ This precludes the use of IHC staining assessment as a standard diagnostic tool to identify LD patient subgroups. 
A.

— Ligand-dependent

— Ligand-independent

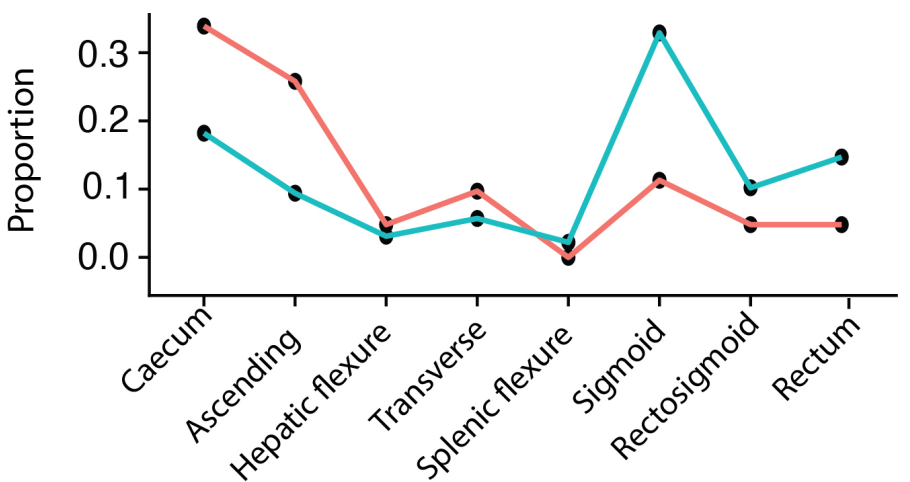

B.

Ligand-dependent
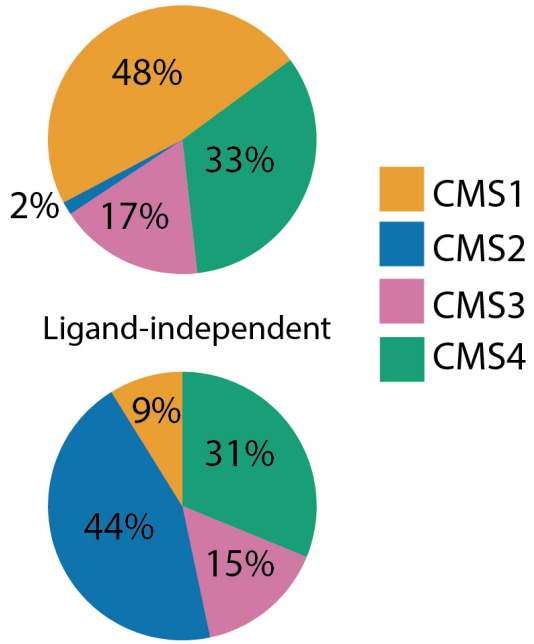

C.
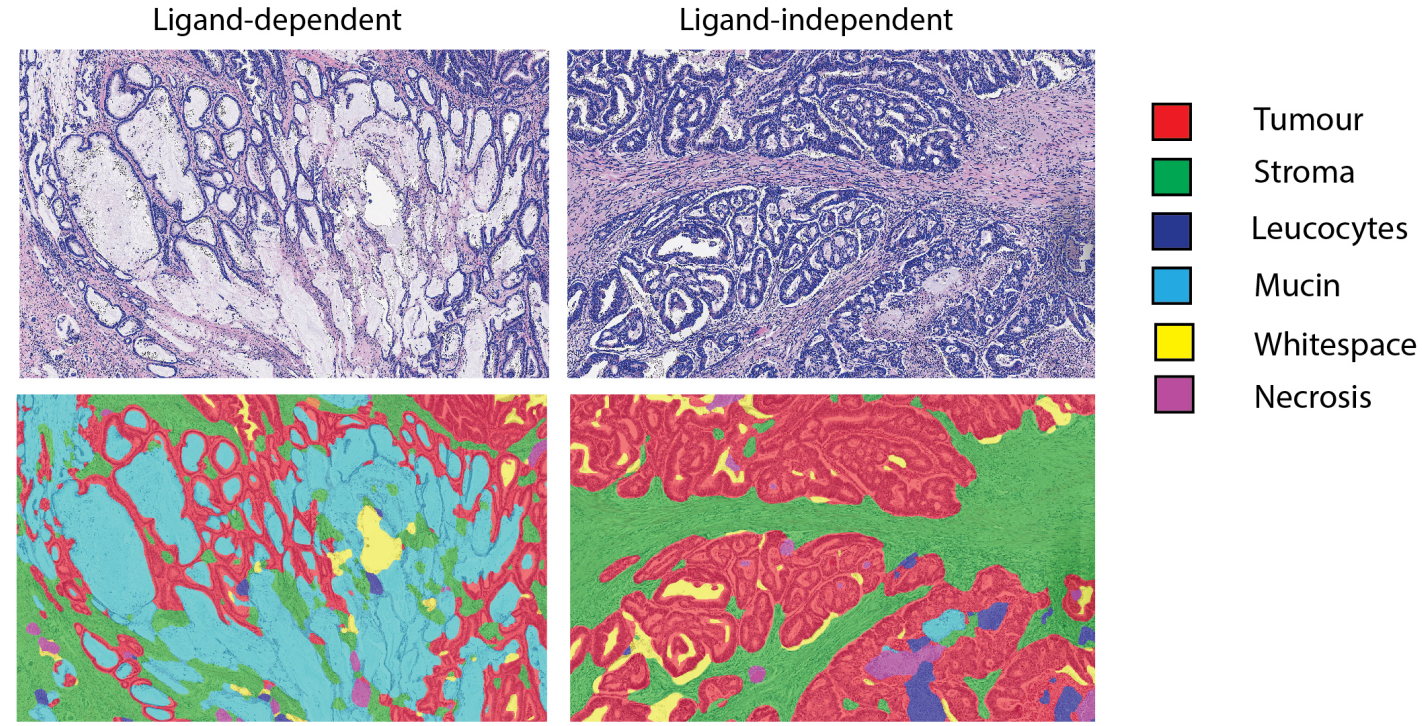

D.

Mucin, manual scoring

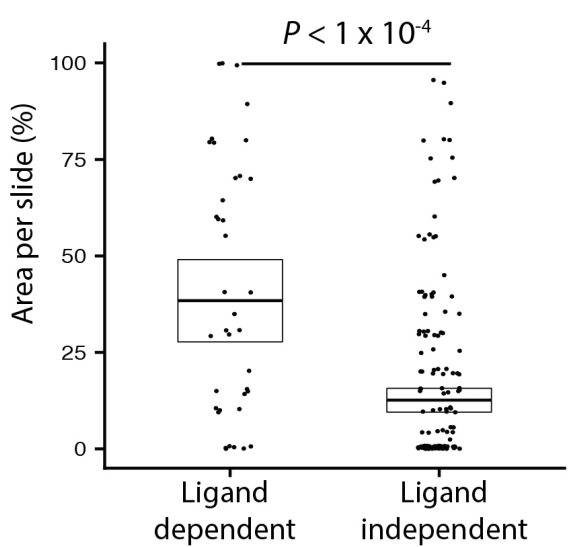

Mucin, automated scoring

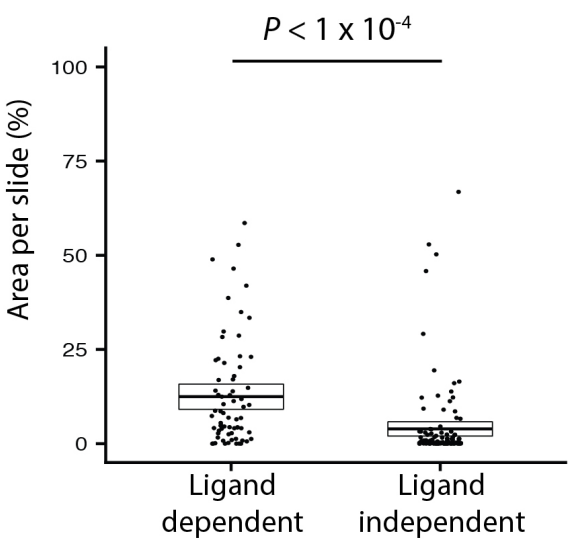

Figure 5 Clinical disease positioning of ligand-dependent and independent tumours. (A) Colonic distribution proportion of ligand-dependent (LD) (red line) and ligand-independent (LI) (blue line) tumours. (B) Consensus molecular subtype (CMS) of LD and LI tumours. (C) Representative images from digital pathology supervised image segmentation of invasive cancer into tissue compartments. (D) Manual and computational scoring of mucin area in LD and LI tumours segregated by molecular ground truth data. 


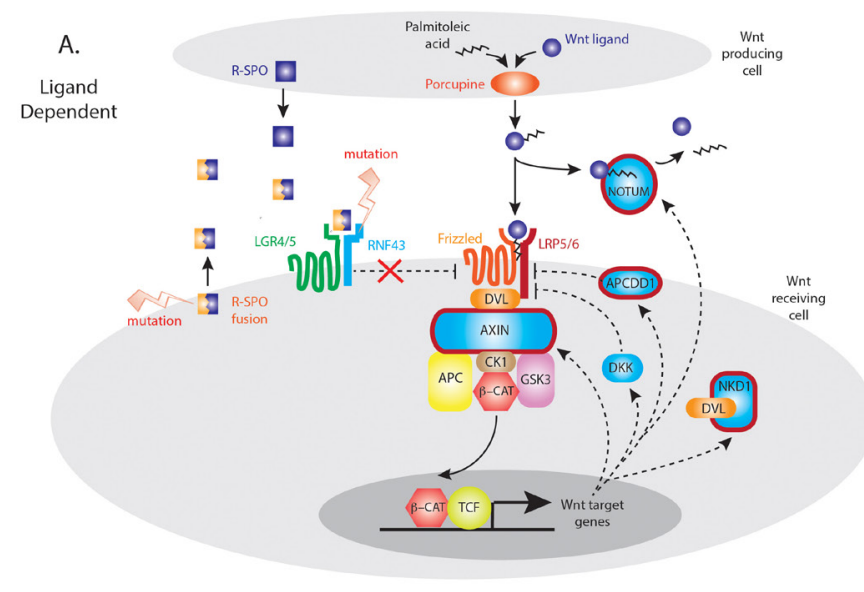

KEY $\longrightarrow$ Negative regulators $\longrightarrow$ Methylation $\triangle M$ Mutation

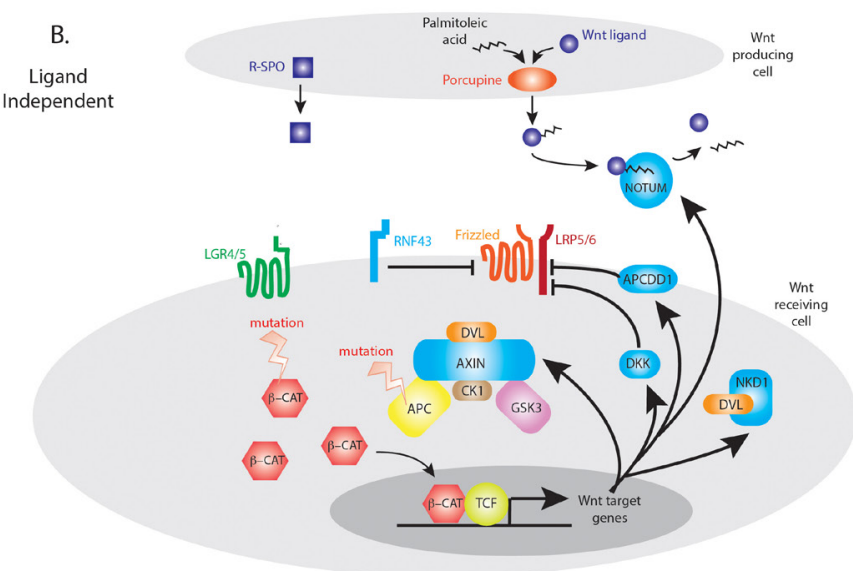

Figure 6 Model of Wnt negative regulation in ligand-dependent (LD) and ligand-independent (LI) tumours. In LD tumours, mutations in the synergistic RSPO axis upregulate Wnt signalling through an otherwise intact canonical Wnt signalling pathway. In this situation, appropriate upregulation of Wnt NRs (blue shapes) could restrain pathological Wnt pathway activity which generates a selective pressure for targeted methylation and epigenetic suppression of key Wnt NR activity (red borders). In LI tumours, downstream mutation in APC and CTNNB1 causes constitutive activation of Wnt intracellular signalling pathways rendering Wnt negative feedback functionally redundant and disconnecting the physiological negative regulator equilibrium.

Across the tumour cohorts in this study, there was a large number of lesions without identifiable Wnt disrupting alterations. This may result partly from missed mutations in retrospective data sets, for example, RNF43 hotspot mutations can be difficult to capture as they are susceptible to microsatellite loci slippage. However, this does not exclude the possibility of rare, novel epithelial (epi) genetic Wnt drivers, or a cell-extrinsic, microenvironmental Wnt source-as seen in RSPO-high lesions. The range of AXIN2 expression seen in the EX lesions here, suggests that AXIN2 could be used to direct the search towards new LD or LI drivers.

Wnt signalling has remained difficult to target in solid tumours, because of developmental and homeostatic importance, pathway complexity and the frequency of non-actionable, signal transduction mutations. Although uncommon, LD tumours represent an opportunity for therapeutic Wnt inhibition and effective small molecule inhibitors are in phase I trials for a range of solid malignancies. ${ }^{26}$ Precision targeted therapies rely on detection of suitable and distinct patient cohorts. Here, we have demonstrated molecular and phenotypic distinction between Wnt disrupted CRC tumour populations and exploited differential Wnt target expression to generate a simple, transcriptome-based biomarker to identify patients with LD tumours for entry into stratified medicine clinical trials.

\section{Author affiliations}

${ }^{1}$ Intestinal Stem Cell Biology Lab, Wellcome Trust Centre Human Genetics, University of Oxford, Oxford, UK

${ }^{2}$ Department of Pathology and Molecular Pathology, University Hospital Zürich,

Zurich, Switzerland

${ }^{3}$ Oxford Colorectal Surgery Department, Nuffield Department of Surgery, Churchill

Hospital, Oxford, Oxfordshire, UK

${ }^{4}$ Translational Gastroenterology Unit, John Radcliffe Hospital, Oxford, UK

${ }^{5}$ Cancer Genetics and Evolution Laboratory, Institute of Cancer and Genomic Sciences, University of Birmingham, Birmingham, West Midlands, UK

${ }^{6}$ Department of Oncology, University of Oxford, Oxford, Oxfordshire, UK

${ }^{7}$ Surgical Research Laboratory, Institute of Cancer \& Genomic Science, University of Birmingham, Birminghaam, United Kingdom

${ }^{8}$ Centre for Cancer Research and Cell Biology, Queen's University Belfast, Belfast, Northern Ireland, UK

${ }^{9}$ Changi General Hospital, Singapore

${ }^{10}$ South Australian Health \& Medical Research Institute \& School of Medicine, The University of Adelaide, Adelaide, South Australia, Australia

Acknowledgements The authors would like to thank Graeme Murray for provision of the rectal cancer biopsies under licence from the Grampian biorepository, to Matt Seymour for use of the FOCUS trial samples and to the MRC FOCUS trial management group.

Contributors SOK, VHK, TM and SJL conceived and designed the project. Experiments were conducted by SOK, VHK, HJSJ, EGV, HD, VP, MAJK, TRML, SLW and DW. Data provision and bioinformatic analysis were carried out by ADB, SOK, RA and ED. Pathology support, tissue provision and intellectual input were from VHK, L-MW, JEE, CC, MBL, PDD and IT. Manuscript written by SOK and SJL.

Funding This work was supported by Wellcome Trust Senior Clinical Research Fellowship (206314/Z/17/Z) and Worldwide Cancer Research grant (16-0042) to $\mathrm{SJL}$, the Stratification in Colorectal Cancer (S:CORT) Consortium, which is funded by the Medical Research Council and Cancer Research UK and the National Institute for Health Research (NIHR) Oxford Biomedical Research Centre. VHK was funded by the Swiss National Science Foundation (P2SKP3_168322/1 and P2SKP3_168322/2). HJSJ was supported by a Bowel Disease Research Foundation (BDRF) research grant. ADB is funded by a Cancer Research UK Advanced Clinician Scientist award (C31641/A23923) and the Wellcome Trust (102732/Z/13/Z). TRML, SLW and DW supported by the NHMRC, Beat Cancer SA \& Cure Cancer Australia. JEE was funded by the National Institute for Health Research (NIHR) Oxford Biomedical Research Centre (BRC). Core funding to the Wellcome Trust Centre for Human Genetics was provided by the Wellcome Trust (090532/Z/09/Z)

Competing interests SJL has received grant income from UCB Pharma. VHK is participant of a patent application co-owned by the Netherlands Cancer Institute (NKI-AVL) and the University of Basel on the assessment of cancer immunotherapy biomarkers by digital pathology and has served as an invited speaker on behalf of Indica Labs. ADB has received grant funding from InCyte for molecular pathology research; speaker fees and travel costs from Illumina UK for immuno-oncology research and consultancy fees from Bristol-Myers-Squibb for immuno-oncology research. All other authors have nothing to disclose.

Patient consent for publication Not required.

Provenance and peer review Not commissioned; externally peer reviewed.

Data availability statement Data relating to transcriptome profiling of relevant genes in polyps cohort and validation cohorts A/B are available online (https:// www.s-cort.org/exploiting-differential-wnt). All other data relevant to the study are referenced, included in the article or uploaded as supplementary information.

Open access This is an open access article distributed in accordance with the Creative Commons Attribution 4.0 Unported (CC BY 4.0) license, which permits others to copy, redistribute, remix, transform and build upon this work for any purpose, provided the original work is properly cited, a link to the licence is given, and indication of whether changes were made. See: https://creativecommons.org/ licenses/by/4.0/.

\section{ORCID iDs}

Sam 0 Kleeman http://orcid.org/0000-0003-1720-8804

Hayley Davis http://orcid.org/0000-0003-2914-4932

Andrew D Beggs http://orcid.org/0000-0003-0784-2967

Susan L Woods http://orcid.org/0000-0002-8955-2017 
Ian Tomlinson http://orcid.org/0000-0003-3037-1470

Philip D Dunne http://orcid.org/0000-0001-9160-283X

\section{REFERENCES}

1 Scoville DH, Sato T, He XC, et al. Current view: intestinal stem cells and signaling. Gastroenterology 2008;134:849-64.

2 Mah AT, Yan KS, Kuo CJ. Wnt pathway regulation of intestinal stem cells. J Physiol 2016;594:4837-47.

3 Shoshkes-Carmel M, Wang YJ, Wangensteen KJ, et al. Subepithelial telocytes are an important source of Wnts that supports intestinal crypts. Nature 2018;557:242-6.

4 Degirmenci B, Valenta T, Dimitrieva S, et al. GLI1-expressing mesenchymal cells form the essential Wnt-secreting niche for colon stem cells. Nature 2018;558:449-53.

5 Farin HF, Jordens I, Mosa MH, et al. Visualization of a short-range Wnt gradient in the intestinal stem-cell niche. Nature 2016;530:340-3.

6 Takada R, Satomi Y, Kurata T, et al. Monounsaturated fatty acid modification of Wnt protein: its role in Wnt secretion. Dev Cell 2006;11:791-801.

7 Yan KS, Janda CY, Chang J, et al. Non-equivalence of Wnt and R-spondin ligands during Lgr5 ${ }^{+}$intestinal stem-cell self-renewal. Nature 2017;545:238-42.

8 Filipovich A, Gehrke I, Poll-Wolbeck SJ, et al. Physiological inhibitors of Wnt signaling. Eur J Haematol 2011;86:453-65.

9 Cancer Genome Atlas Network. Comprehensive molecular characterization of human colon and rectal cancer. Nature 2012;487:330-7.

10 Giannakis M, Hodis E, Jasmine Mu X, et al. RNF43 is frequently mutated in colorectal and endometrial cancers. Nat Genet 2014;46:1264-6.

11 Seshagiri S, Stawiski EW, Durinck S, et al. Recurrent R-spondin fusions in colon cancer. Nature 2012;488:660-4.

12 Lamlum $H$, llyas M, Rowan A, et al. The type of somatic mutation at APC in familial adenomatous polyposis is determined by the site of the germline mutation: a new facet to Knudson's 'two-hit' hypothesis. Nat Med 1999;5:1071-5.
13 Sekine S, Yamashita S, Tanabe T, et al. Frequent PTPRK-RSPO3 fusions and RNF43 mutations in colorectal traditional serrated adenoma. J Pathol 2016;239:133-8.

14 Madan B, Ke Z, Harmston N, et al. Wnt addiction of genetically defined cancers reversed by PORCN inhibition. Oncogene 2016;35:2197-207.

15 Hashimoto T, Yamashita S, Yoshida H, et al. WNT pathway gene mutations are associated with the presence of dysplasia in colorectal sessile serrated adenoma/ polyps. Am J Surg Pathol 2017;41:1188-97.

16 Sekine S, Ogawa R, Hashimoto T, et al. Comprehensive characterization of RSPO fusions in colorectal traditional serrated adenomas. Histopathology 2017;71:601-9.

17 Hoadley KA, Yau C, Hinoue T, et al. Cell-of-origin patterns dominate the molecular classification of 10,000 tumors from 33 types of cancer. Cell 2018;173:291-304.

18 Isella C, Terrasi A, Bellomo SE, et al. Stromal contribution to the colorectal cancer transcriptome. Nat Genet 2015;47:312-9.

19 Muto Y, Maeda T, Suzuki K, et al. DNA methylation alterations of AXIN2 in serrated adenomas and colon carcinomas with microsatellite instability. BMC Cancer 2014; $14: 466$.

20 Grasso CS, Giannakis M, Wells DK, et al. Genetic mechanisms of immune evasion in colorectal cancer. Cancer Discov 2018;8:730-49.

21 Koinuma K, Yamashita Y, Liu W, et al. Epigenetic silencing of AXIN2 in colorectal carcinoma with microsatellite instability. Oncogene 2006;25:139-46.

22 Toyota M, Ahuja N, Ohe-Toyota M, et al. CpG island methylator phenotype in colorectal cancer. Proc Natl Acad Sci U S A 1999;96:8681-6.

23 Storm EE, Durinck S, de Sousa e Melo F, et al. Targeting PTPRK-RSPO3 colon tumours promotes differentiation and loss of stem-cell function. Nature 2016:529:97-100.

24 Longerich T, Endris V, Neumann 0, et al. RSPO2 gene rearrangement: a powerful drive of $\beta$-catenin activation in liver tumours. Gut 2019:68:1287-96.

25 Gao C, Xiao G, Hu J. Regulation of Wnt/ $\beta$-catenin signaling by posttranslational modifications. Cell Biosci 2014:4

26 ClinicalTrials.gov. Study to evaluate the safety and tolerability of RXC004 in advanced malignancies. Available: https://clinicaltrials.gov/ct2/show/NCT03447470 [Accessed 17 May 2018]. 